\title{
Sensitivity to longitudinal vector boson scattering in semileptonic final states at the HL-LHC
}

\author{
Jennifer Roloff॰, Viviana Cavaliere $\odot$, and Marc-André Pleier® \\ Physics Department, Brookhaven National Laboratory, Upton, New York 11973-5000, USA \\ Lailin Xu® \\ Department of Modern Physics and State Key Laboratory of Particle Detection and Electronics, \\ University of Science and Technology of China, Hefei 230026, China
}

(Received 31 July 2021; accepted 29 September 2021; published 5 November 2021)

\begin{abstract}
Longitudinal vector boson scattering provides an important probe of electroweak symmetry breaking, bringing sensitivity to physics beyond the Standard Model as well as constraining properties of the Higgs boson. It is a difficult process to study due to the small production cross section and challenging separation of the different polarization states. We study the sensitivity to longitudinal $W V$ vector boson scattering at the high-luminosity Large Hadron Collider in semi-leptonic final states. While these are characterized by larger background contributions compared to fully leptonic final states, they benefit from a higher signal cross section due to the enhanced branching fraction. We determine the polarization through full reconstruction of the event kinematics using the $W$ boson mass constraint and through the use of jet substructure. We show that with these techniques sensitivities around three standard deviations at the HL-LHC are achievable, which makes this channel competitive with its fully leptonic counterparts.
\end{abstract}

DOI: $10.1103 /$ PhysRevD.104.093002

\section{INTRODUCTION}

The study of longitudinal vector boson scattering (VBS) processes remains a long-standing, yet unattained, milestone of high energy physics [1]. The increase of the scattering amplitude of longitudinal vector bosons with center-of-mass energy in absence of a Higgs boson, eventually violating unitarity [2-4], led to the formulation of the "no-lose theorem" for colliders of sufficiently high energies, postulating that either a Higgs boson or some new physics beyond the Standard Model (SM) be found [5]. The discovery of a Higgs-like boson [6,7] at the Large Hadron Collider (LHC) in 2012 heralded the first step toward the study of the delicate interplay at work in longitudinal VBS. While the properties of this Higgs boson thus far were found to be consistent with SM expectations [8-11], even small deviations in its vector boson couplings could give rise to an increase of the scattering amplitude of longitudinal vector bosons with center-of-mass energy, which in turn renders longitudinal VBS a sensitive probe for anomalous Higgs couplings [12,13]. Furthermore, a rich portfolio of models beyond the SM predicts enhancements

Published by the American Physical Society under the terms of the Creative Commons Attribution 4.0 International license. Further distribution of this work must maintain attribution to the author(s) and the published article's title, journal citation, and DOI. Funded by SCOAP ${ }^{3}$. of VBS production through extended Higgs sectors or other new resonances [14-19].

While experimentally VBS processes offer the distinct signature of a forward/backward pair of jets $(j)$ which are well-separated in rapidity ("tagging" jets) and exhibit a large invariant dijet mass, as well as the decay products of the produced vector bosons, their measurement is challenging due to small cross sections. Both ATLAS and CMS have by now established VBS processes involving massive vector bosons $(V=W, Z)$ in fully leptonic decay modes, successfully separating the desired purely electroweak production from strong (QCD-induced) $V V j j$ production and other background processes [20-25]. Studies of semileptonic VBS final states where one vector boson decays hadronically-albeit benefitting from the large $V$ hadronic branching fraction compared to the leptonic decays-thus far were unable to firmly establish the SM signal due to increased background levels, but have proven to provide excellent sensitivity to anomalous couplings [26-28]. Observation of the semileptonic $W V j j$ VBS process is expected to be achievable at the LHC with an integrated luminosity of $300 \mathrm{fb}^{-1}$ at $\sqrt{s}=14 \mathrm{TeV}$ [29].

Measuring longitudinal VBS processes is further complicated by the difficulty of separating longitudinal states from transverse ones. First studies of $V V$ and $V V j j$ LHC data in fully leptonic vector boson decay modes explore the possibilities of extracting cross sections for longitudinally polarized vector bosons, but their sensitivity is still 
TABLE I. Overview of the simulated MC samples.

\begin{tabular}{lccc}
\hline \hline Process & Accuracy & Cross section [fb] & Number of events \\
\hline$W^{ \pm} W^{\mp} j j$ & LO & 0.325 & $2.7 \mathrm{e} 6$ \\
$W Z j j$ & LO & 0.114 & $3.9 \mathrm{e} 5$ \\
$W^{ \pm} W^{ \pm} j j$ & OO & 0.114 & $5.89 \mathrm{e} 5$ \\
$W+$ jets & LO & 1185 & $1.09 \mathrm{e} 7$ \\
$t \bar{t}$ & NLO & 374 & $8.64 \mathrm{e} 6$ \\
\hline \hline
\end{tabular}

insufficient in the currently available datasets to access longitudinal VBS [30,31]. Projections to the HighLuminosity LHC (HL-LHC), providing proton-proton collisions at a center-of-mass energy of $\sqrt{s}=14 \mathrm{TeV}$ with an integrated luminosity of $3000 \mathrm{fb}^{-1}$, show $W^{ \pm} W^{ \pm} j j$ as the most promising channel to establish longitudinal VBS in fully leptonic vector boson decay modes, but none of the $W^{ \pm} W^{ \pm} j j, W Z j j$ and $Z Z j j$ processes studied is predicted to reach a significance of 3 standard deviations at a single experiment [32-37]. Using more sophisticated analysis techniques such as deep machine learning, the sensitivity for longitudinal VBS can be significantly increased, as demonstrated for $W^{ \pm} W^{ \pm} j j$ in [38] for the leptonic decay channel.

The sensitivity to longitudinal VBS in semi-leptonic final states has been explored much less. While some studies exist for $\sqrt{s}=13 \mathrm{TeV}$ [39] or $\sqrt{s}=27 \mathrm{TeV}$ [40], the sensitivity to longitudinal VBS in semileptonic final states at the HL-LHC has not been assessed-a gap that this paper is addressing. We focus on the $W V j j$ channel where one $W$ boson decays into a charged lepton (an electron or muon, denoted by $\ell$ ) and an (anti-) neutrino $\nu$, while the other massive vector boson $V$ is considered to decay into a pair of quarks which we require to be reconstructed as a merged, large-radius jet $(J)$, leading to an $\ell \nu J j j$ final state. To enable full reconstruction of the event kinematics, the neutrino four-vector is recreated by imposing a $W$ boson mass constraint in the lepton neutrino system. The expected significant impact of pileup at the HL-LHC is mitigated by using track-based observables, and jet substructure techniques are deployed to improve $V$ boson reconstruction. As both the resolved $W V j j$ channel (where the hadronic $V$ decay is reconstructed via two separate small-radius jets) and $Z V j j$ semileptonic final states will contribute to establishing longitudinal VBS in semileptonic final states, our results can be seen as a lower limit on the expected sensitivity at the HL-LHC.

\section{SIMULATION SAMPLES}

Electroweak $W V j j$ production includes contributions from the $W^{ \pm} W^{\mp} j j, W^{ \pm} W^{ \pm} j j$ and $W Z j j$ VBS processes, which are modeled with MadGraph5_aMC@NLO 2.7.3 [41], interfaced to PYTHIA 8.243 [42] for parton showering and hadronization. These samples are generated with two on-shell vector bosons, with one $W$ boson decaying leptonically $(W \rightarrow \ell \nu$ ), and the other massive vector boson decaying hadronically. The contribution from triboson processes is also included, but negligible in the phase space studied (see Sec. III). Four different polarization states are produced at leading order in QCD: both bosons are longitudinally polarized $\left(W_{L} V_{L}\right)$, both transversely polarized $\left(W_{T} V_{T}\right)$, or a mixture $\left(W_{T} V_{L}\right.$ and $\left.W_{L} V_{T}\right)$. These polarized samples are simulated with the helicity eigenstates defined in the $W V$ center-of-mass reference frame [43]. For this analysis focused on $W_{L} V_{L}$ production, the signal is referred to as VBS $W_{L} V_{L}$, while the other polarization states $\left(W_{T} V_{T}, W_{T} V_{L}\right.$, and $\left.W_{L} V_{T}\right)$ are referred to as the VBS $W V$ background.

The main background contributions for this analysis are the production of a $W$ boson in association with jets and top-quark pair production. The $W+$ jets samples are simulated using CKKW-L merging $[44,45]$ with up to four partons at leading order in QCD using MadGraph5_aMC@NLO 2.7.3. The top-quark pair production sample is generated using MadGraph5_aMC@NLO 2.7.3 at next-to-leading order in QCD, and the top quarks are decayed using MADSPIN [46] in order to preserve the spin correlations for top-quark production and decay. PYTHIA 8.243 is used for parton showering and hadronization for all background samples. The contribution of QCD-induced $V W \mathrm{jj}$ processes in our signal region is found to be a factor of 100 smaller than the $W+$ jets background, and a factor of two smaller than the VBS $V W$ EW-induced backgrounds, and is not considered further in our studies.

A parton level event filter of $H_{T}>200 \mathrm{GeV}$ is used to enhance the statistical power of the Monte Carlo (MC) samples in the phase space studied, where $H_{T}$ is the sum of the transverse momentum $\left(p_{T}\right)$ of all partons. Leptons and partons are also required to satisfy $p_{T}>$ $10 \mathrm{GeV}$ at the generator level. This filter is found to be fully efficient for the concerned phase space in this study (see Sec. III). Table I summarizes the simulated MC samples. The number of events generated in particular for the background processes is driven by the requirement that there be no empty bins in the discriminant used to determine the analysis sensitivity (see Sec. V), hence avoiding any extrapolations across empty bins. All signal and background processes are reconstructed using a generic detector in the Delphes simulation framework [47], modeled after the ATLAS detector in the HL-LHC [48].

\section{EVENT SELECTION}

Events from VBS $W V$ production exhibit several distinct characteristics which may be used in the event selection. In the semileptonic decay, the event contains one lepton and missing transverse momentum $E_{\mathrm{T}}^{\text {miss }}$ from the leptonic $W$ boson decay, and either two jets or a large-radius jet from 
TABLE II. The event selection.

\begin{tabular}{ll}
\hline \hline Object & \multicolumn{1}{c}{ Selection } \\
\hline Lepton & $p_{T, \ell}>20 \mathrm{GeV}$ \\
& $\left|\eta_{\ell}\right|<4.0$ \\
& No other leptons with $p_{T, \ell}>7 \mathrm{GeV}$ \\
& $p_{T, J}>200 \mathrm{GeV}$ \\
& $\left|\eta_{J}\right|<4.0$ \\
Large-R jet $J$ & $40 \mathrm{GeV}<m_{J}<180 \mathrm{GeV}$ \\
& $E_{\mathrm{T}}^{\text {miss }}>80 \mathrm{GeV}$ \\
& $p_{T, j}>30 \mathrm{GeV}$ \\
& $\left|\eta_{j}\right|<4.0$ \\
$E_{\mathrm{T}}^{\text {miss }}$ & $\Delta R_{j, J}>1.4, \Delta R_{j, \ell}>0.4$ \\
tagging jets $\left(j_{1}, j_{2}\right)$ & $m_{j_{1} j_{2}}>800 \mathrm{GeV}$ \\
& $\eta_{j_{1}} \cdot \eta_{j_{2}}<0$ \\
& No b-tagged jets in the event \\
& with $\Delta R_{J, j}>1.0$ \\
\hline \hline
\end{tabular}

the hadronic $V$ decay. In addition to the $V$ boson decay products, there are two forward jets from the VBS production, which are referred to as the "tagging" jets.

As detailed in Table II, a loose selection is applied to the events, based on the expected reconstruction capabilities at the HL-LHC [49]. In order to select the leptonically decaying $W$ boson, each event is required to have an electron or muon with $p_{T}>20 \mathrm{GeV}$ and pseudorapidity $\left|\eta_{\ell}\right|<4.0$, and to contain no other leptons with $p_{T}>7 \mathrm{GeV}$.

Our study focuses on the case where the hadronically decaying $V$ boson candidate can be reconstructed as a single large-radius jet $J$. The inclusion of the resolved case where the decay products are reconstructed as separate jets would improve the significance of these results, but is not considered due to the combinatoric challenges in assigning tagging- and $V$-decay jets. Jets are clustered with FASTJET [50] using the anti- $k_{t}$ algorithm [51] with radius parameter of $R=1.0$, using "particle flow objects" as inputs to the jet reconstruction algorithm. These particle flow inputs combine information from the tracker and calorimeter in order to provide better resolution for object reconstruction. The large-radius jets are groomed using the soft-drop grooming algorithm, with $\beta=1.0$, and $z_{\text {cut }}=0.1$ [52] in order to reduce effects due to multiple simultaneous $p p$ collisions (pileup) and the underlying event, and to improve sensitivity of the $V$ boson reconstruction. The large-radius jet is required to have $p_{T}>200 \mathrm{GeV}$ in order to reconstruct both decay products within a single jet, and $|\eta|<4$.0, and it is required to be isolated from the lepton by $\Delta R_{\ell, J}>1.0$. If multiple large-radius jets are reconstructed, the highest- $p_{T}$ jet is selected. After the jet is selected, its mass is required to satisfy $40<m_{J}<180 \mathrm{GeV}$.

Missing transverse momentum is reconstructed as the negative sum of the transverse momentum of all particle-flow objects within $|\eta|<5$.0, and is required to be greater than $80 \mathrm{GeV}$ to reduce QCD background contributions.
The two quarks produced in the VBS production are reconstructed using small-radius $(R=0.4)$ jets. These jets are required to have $p_{T}>30 \mathrm{GeV}$ and $|\eta|<4.0$, and they must be isolated from the selected large-radius jet by $\Delta R>1.4$, and from the lepton by $\Delta R>0.4$. The two jets that maximize the dijet invariant mass $\left(m_{j_{1} j_{2}}\right)$ and are in opposite hemispheres $\left(\eta_{j_{1}} \cdot \eta_{j_{2}}<0\right)$ are identified as the tagging jets, and events are required to have $m_{j_{1} j_{2}}>$ $800 \mathrm{GeV}$ to reduce the background contributions. To lower contributions from top-quark pair production, the event is required to have no b-tagged jets outside of the selected large- $\mathrm{R}$ jet.

\section{A. $V$ boson reconstruction}

After above event selection, both of the $V$ bosons are reconstructed at detector level. The large-radius jet serves as a proxy for the hadronically decaying $V$ boson. Since the jet has been groomed with the soft-drop algorithm, the two associated subjets which pass the soft-drop condition are natural proxies for the decay products of the $V$ boson.

The leptonically decaying $W$ boson is fully reconstructed using the lepton and $E_{\mathrm{T}}^{\text {miss }}$, using the $W$ boson mass to fully constrain the kinematics, with the assumption that the $E_{\mathrm{T}}^{\text {miss }}$ arises solely from the neutrino. The neutrino transverse momentum is taken to be the $E_{\mathrm{T}}^{\text {miss }}$, and the longitudinal component is solved for by assuming the $W$ boson is onshell, and that the charged lepton is massless. The result of this is a second order polynomial with two solutions. In cases where there are no real solutions, the longitudinal momentum is taken to be the real component of the solution. In cases where there are two real solutions, the solution with the smaller longitudinal momentum is taken, which produces the correct result in around $65 \%$ of generated events.

\section{B. Polarization}

In the $V$ boson rest frame, the decay products of the $V$ boson will be back-to-back, and can be characterized based on the angle $\theta^{*}$ between the $V$ boson direction and the decay product direction. The $V$-boson differential cross section depends on the polarization fractions as

$$
\begin{aligned}
\frac{d \sigma}{d \cos \theta^{*}} \propto & \frac{3}{8} f_{-}\left(1 \mp \cos \theta^{*}\right)^{2}+\frac{3}{8} f_{+}\left(1 \pm \cos \theta^{*}\right)^{2} \\
& +\frac{3}{4} f_{L}\left(1-\cos ^{2} \theta^{*}\right), \quad \text { for } \mathrm{W}^{ \pm}
\end{aligned}
$$

where $f_{-}, f_{+}$, and $f_{L}$ are the fractions of events where the $V$ boson polarization is $-1,+1$, and 0 , respectively. Similarly, in the laboratory frame, the decay products for the longitudinally polarized $V$ bosons will tend to be more balanced in $p_{T}$, and less balanced for transversely polarized $V$ bosons. Consequently, the momentum balance of the 
leptonic decay products, or $z_{g, \ell}=p_{T, \ell} / p_{T, W}$, and $\cos \left(\theta^{*}\right)_{\ell}$ are sensitive variables to the $W$ boson polarization.

Similar variables may be defined for the hadronic case as well, using the large-R jet and its two subjets as proxies for the hadronically decaying $V$ boson and its decay products.

Using MC generator truth information, the decay products are distinguishable as quark $(q)$ and anti-quark $(\bar{q})$, and we can use for example $\cos \left(\theta^{*}\right)_{q}$ (defined using the angle between the $V$ boson direction and the quark $q$ from the $V$ boson decay), and $z_{g, q}=p_{T, q} / p_{T, V}$ as polarizationsensitive observables without introducing a kinematical bias, albeit not reconstructable in data. At detector level, the two subjets are only distinguishable by their kinematics, and denoting the leading $p_{T}$ subjet $q_{1}$ and the subleading $p_{T}$ subjet $q_{2}$, we can define e.g., $\cos \left(\theta^{*}\right)_{q_{1}}$ and $z_{g, q_{1}}=$ $p_{T, q_{1}} / p_{T, V}$ accordingly. While this biases the kinematics (as illustrated in Fig. 1), these observables are accessible with the detector.

To validate the assumption that the subjets are good proxies for the $V$ boson decay products, above observables-both using $q$ and using $q_{1}$-are studied with a few additional requirements. To reduce the contributions of events where the $V$ boson decay products are not contained within a single large-radius jet, the $V$ boson is required to be matched to the
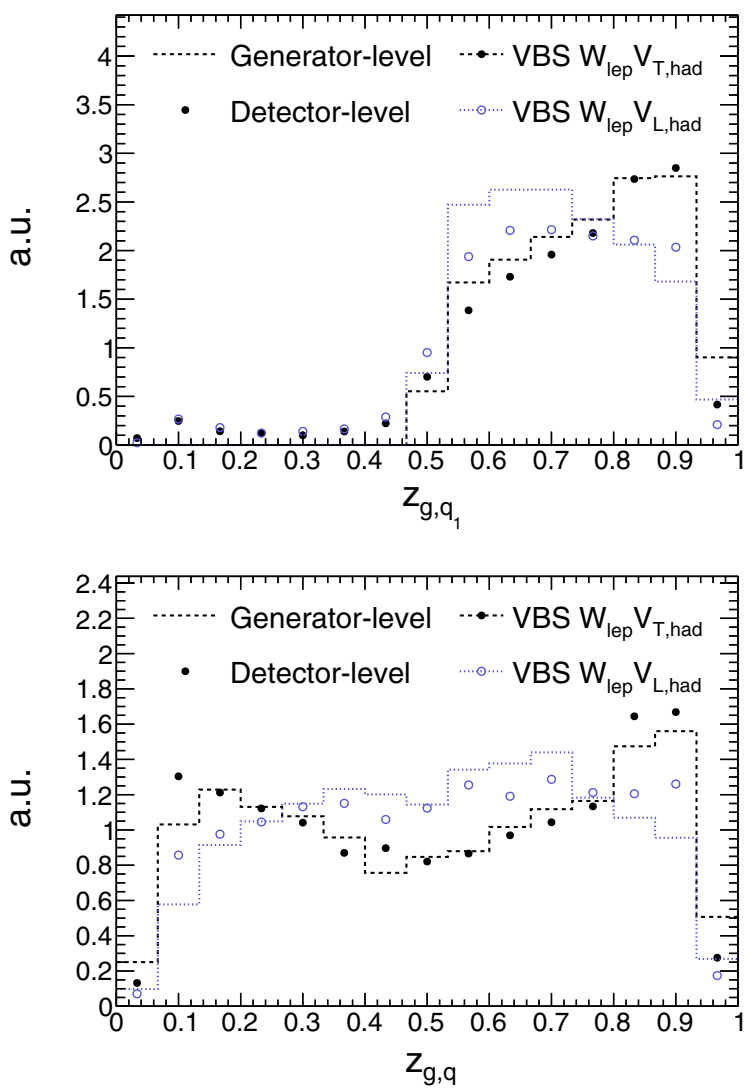

selected large-radius jet with $\Delta R_{V, J}<0.4$. The subjets are ordered with the same $\eta$-ordering as the generator-level decay products, to avoid any bias from a direct matching of the subjets and the generator-level decay products. A comparison of the generator-level and detector-level distributions for $\cos \theta^{*}$ and $z_{g}$ is shown for the hadronically decaying $V$ boson in Fig. 1, which demonstrates that the subjets indeed are good proxies for the $V$ boson decay products, and can be used to distinguish between the different polarization states of the $V$ boson.

At the HL-LHC, reconstruction is complicated by the impact of radiation from pileup on these observables. In particular, jet substructure is sensitive to the wide-angle, low- $p_{T}$ particles associated with pileup. Particle flow objects include calorimeter measurements, where pileup is difficult to separate from the hard-scatter collision, while for tracks, pileup may be removed based on the primary vertex association. In order to mitigate their pileup sensitivity, jet substructure observables can be calculated using tracks as inputs rather than using particle-flow objects. To reconstruct these track-based observables, tracks are associated to a large- $\mathrm{R}$ jet using a $\Delta R<1.0$ matching. These tracks are then clustered and groomed using the same algorithms as the particle-flow jets. Consequently, each
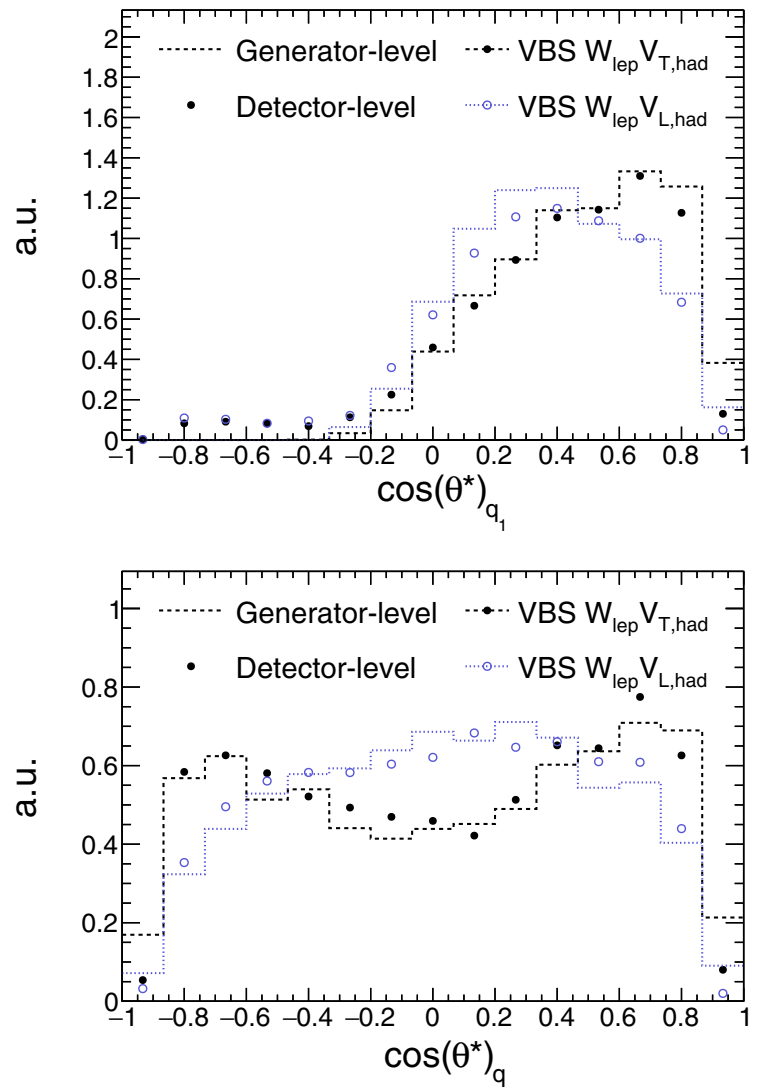

FIG. 1. Comparison of (left) the $p_{T}$ balance of the hadronically decaying $V$ decay products, and (right) the $\cos \left(\theta^{*}\right)$ distribution for generator and detector level reconstruction for longitudinally and transversely polarized $V$ bosons, using (top) the leading $p_{T}$ subjet $q_{1}$, and (bottom) the quark $q$ from the $V$ boson decay. 

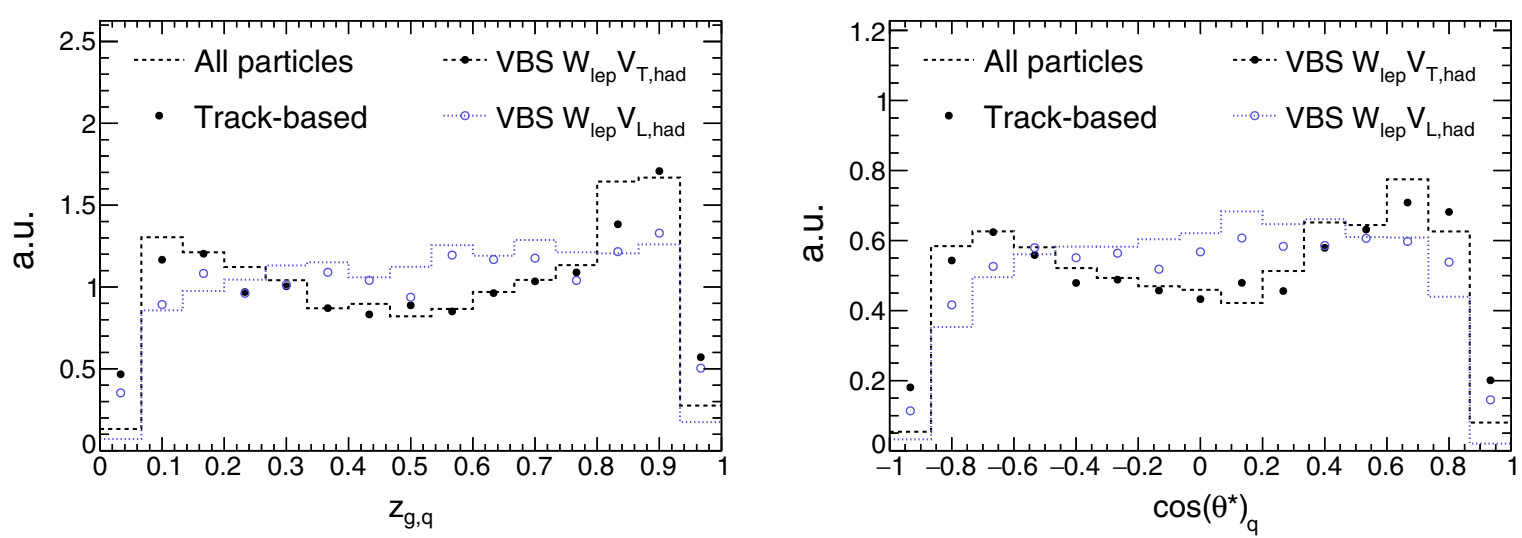

FIG. 2. Comparison of (left) The $p_{T}$ balance of the hadronically decaying $V$ decay products, and (right) the $\cos \left(\theta^{*}\right)$ distribution for particle-flow-based and track-based substructure reconstruction for longitudinally and transversely polarized $V$ bosons.

substructure observable may be calculated using either the particle-flow constituents of the jet, or the groomed tracks associated to the jet. As illustrated in Fig. 2, track-based observables are able to capture similar information as the particle-flow observables and hence are used in our analysis from here on for substructure observables whose pileup sensitivity has not been studied in detail, namely any substructure observables which are not the jet mass or ratios of energy correlation functions such as $d_{2}$ [53].

Since the leptonically decaying $W$ boson is fully reconstructed, it is also possible to define similar observables using the lepton and the reconstructed $W$ boson. The corresponding results are shown for events with particlelevel $E_{\mathrm{T}}^{\text {miss }}>80 \mathrm{GeV}$ in Fig. 3, illustrating that the reconstructed $W$ boson decay behaves similarly to the generator-level $W$ boson. In the transversely polarized case, the lepton tends to have a $p_{T}$ smaller than the neutrino. This is a result of the $E_{\mathrm{T}}^{\text {miss }}$ cut, which biases the relative momenta of the $W$ decay products.

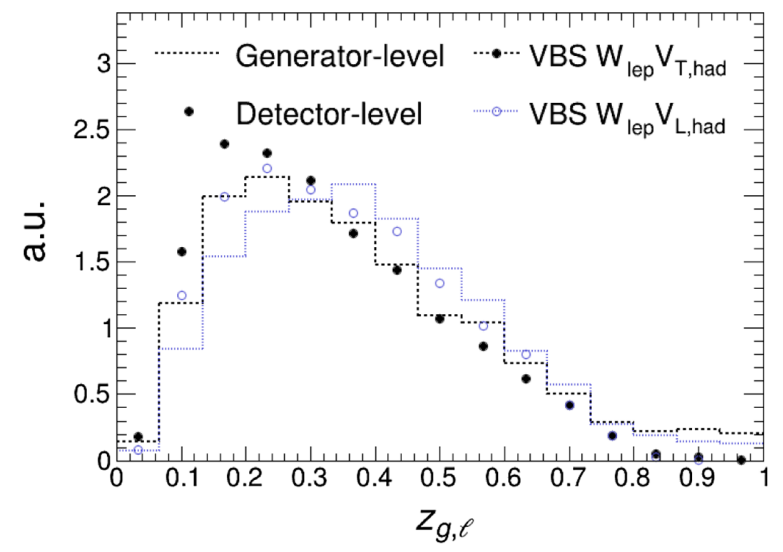

\section{SIGNAL EXTRACTION}

Three main background processes need to be considered to extract the longitudinal VBS signal: $W+$ jets and topquark pair production, as well as the VBS non- $W_{L} V_{L}$ polarization states. To illustrate the initial signal-to-background ratio, the event yields of signal and background for $3000 \mathrm{fb}^{-1}$ of data after applying the event selection are shown in Fig. 4 for several observables. No single observable offers sufficient background reduction on its own, but by combining multiple observables in a neural network, the background reduction can be significantly improved.

Each different background has unique characteristics which may be used to distinguish it from the $W_{L} V_{L}$ signal process:

(i) The background VBS $W V$ events have a similar topology, but differ for variables sensitive to the polarization states.

(ii) The $W+$ jets background does not contain a hadronically decaying $V$ boson, and the tagging jets will tend to be more central.

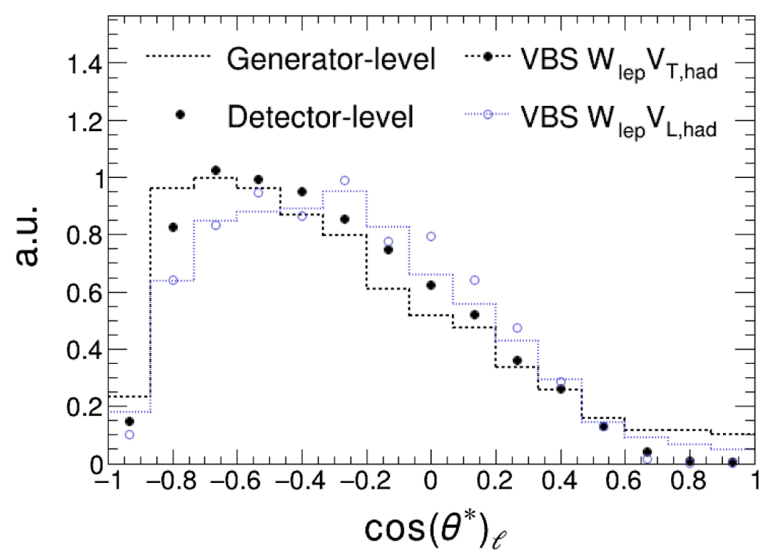

FIG. 3. Comparison of (left) the $p_{T}$ balance of the leptonically decaying $W$ decay products, and (right) the $\cos \left(\theta^{*}\right)$ distribution for generator and detector level reconstruction for longitudinally and transversely polarized $W$ bosons. 

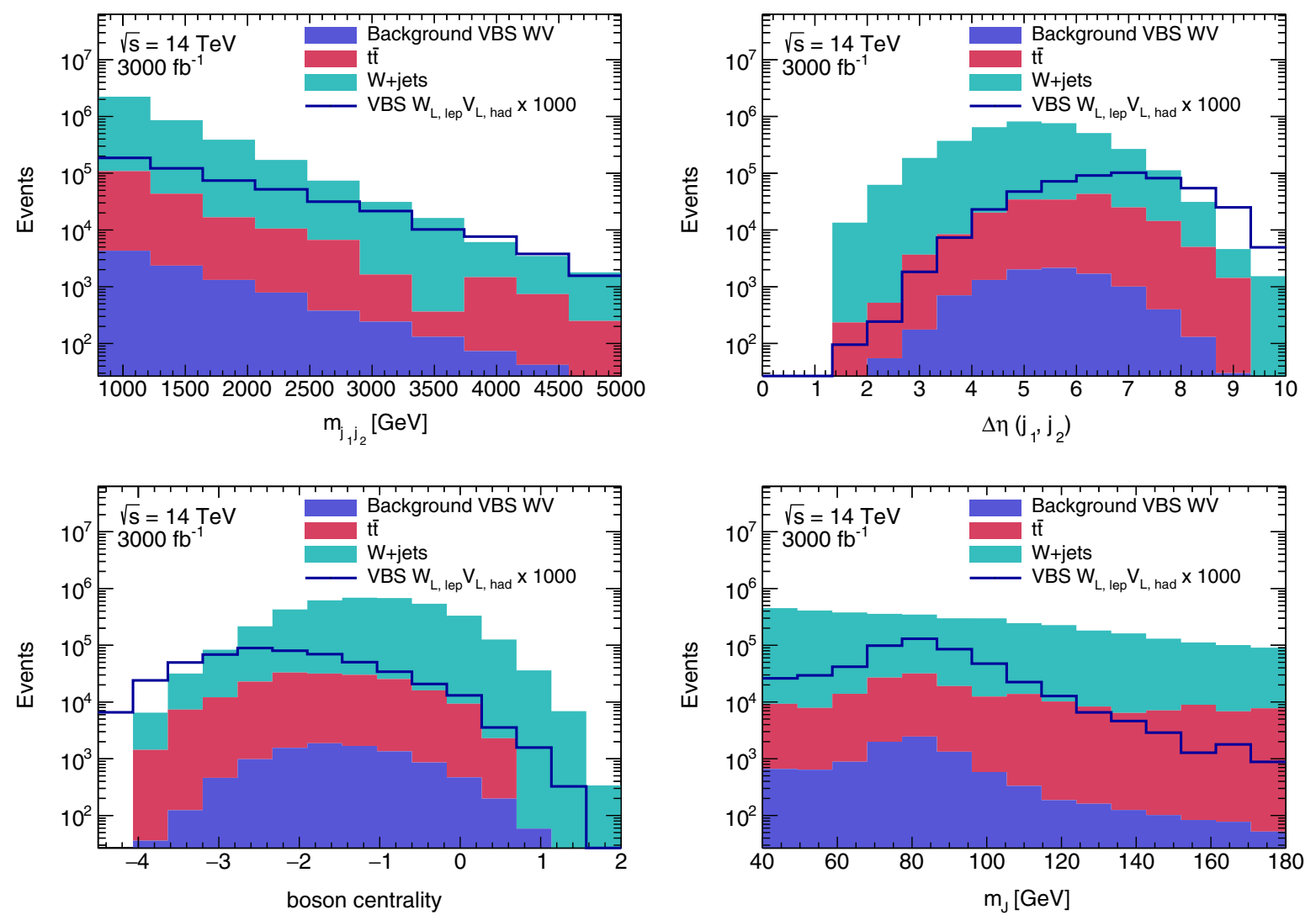

FIG. 4. Kinematic distributions of the $W_{L} V_{L}$ signal compared to the three main background processes after the event selection for an integrated luminosity of $3000 \mathrm{fb}^{-1}$.

(iii) The top-quark pair production background contains a hadronically decaying $W$ boson, and will tend to have more (heavy flavor) jets in the event.

Because of this, it is difficult to train a tagger to effectively distinguish between the $W_{L} V_{L}$ events and all background processes. In order to improve analysis sensitivity, a multiclass tagger is trained to identify four different classes of events: the signal (VBS $W_{L} V_{L}$ ), the other (background) polarization states of VBS $W V, W+$ jets, and top-quark pair production.

The multiclass tagger is trained using the TMVA [54] implementation of multiclass deep neural network (DNN) based on a multilayer perceptron with one hidden layer and 17 neurons. Twelve variables, listed in Table III, are used as inputs into a multiclass DNN tagger. The distributions of these input variables are shown in Fig. 5 for both signal and background, with the pseudorapidity difference between the two tagging jets $\Delta \eta\left(j_{1}, j_{2}\right)$ yielding the best single-variable signal discrimination. For reference purposes, we compare signal extraction based on $\Delta \eta\left(j_{1}, j_{2}\right)$ alone (while additionally requiring the jet mass to be $60 \mathrm{GeV}<m_{J}<100 \mathrm{GeV}$, and $d_{2, J}<1.5$ to further reduce background contributions), with our DNN performance. The event yield for the DNN tagger score compared to $\Delta \eta\left(j_{1}, j_{2}\right)$ is shown for the signal and background events in Fig. 6, illustrating that the discrimination power of the
DNN score for the signal class is significantly better than the discrimination power of the most important input variable to the tagger $\Delta \eta\left(j_{1}, j_{2}\right)$. This is expected, as the DNN is able to better separate the signal events from the

TABLE III. The variables used in the signal tagger.

\begin{tabular}{ll}
\hline \hline Variable & \multicolumn{1}{c}{ Description } \\
\hline$p_{T, \ell}$ & $p_{T}$ of the charged lepton from the $W$ boson \\
$\eta_{\ell}$ & Pseudorapidity of the charged lepton from \\
the $W$ boson & $p_{T}$ of the reconstructed leptonically \\
& decaying $W$ boson \\
$p_{T, W(\ell \nu)}$ & Mass of the large-R jet \\
$m_{J}$ & Ratio of three-point to two-point \\
$d_{2, J}$ & energy correlation functions \\
$r_{g, J}$ & Angular separation between the two subjets \\
$p_{T, \mathrm{WV}}$ & $p_{T}$ of the diboson system \\
$m_{\mathrm{WV} j_{1} j_{2}}$ & Mass of the $W V j_{1} j_{2}$ system \\
Boson centrality & Min $\left(\Delta \eta_{-}, \Delta \eta_{+}\right)$, with \\
& $\Delta \eta_{-}=$min $\left[\eta_{V_{\text {had }}}, \eta_{W_{\text {lep }}}\right]-\min \left[\eta_{j_{1}}, \eta_{j_{2}}\right]$ \\
& $\Delta \eta_{+}=\max \left[\eta_{V_{\text {had }}}, \eta_{W_{\text {lep }}}\right]-\max \left[\eta_{j_{1}}, \eta_{j_{2}}\right]$ \\
$p_{T, j_{1}}$ & $p_{T}$ of the leading tagging jet \\
$\eta_{j_{1}}$ & $\eta$ of the leading tagging jet \\
$\Delta \eta\left(j_{1}, j_{2}\right)$ & Pseudorapidity difference between the \\
& two tagging jets
\end{tabular}



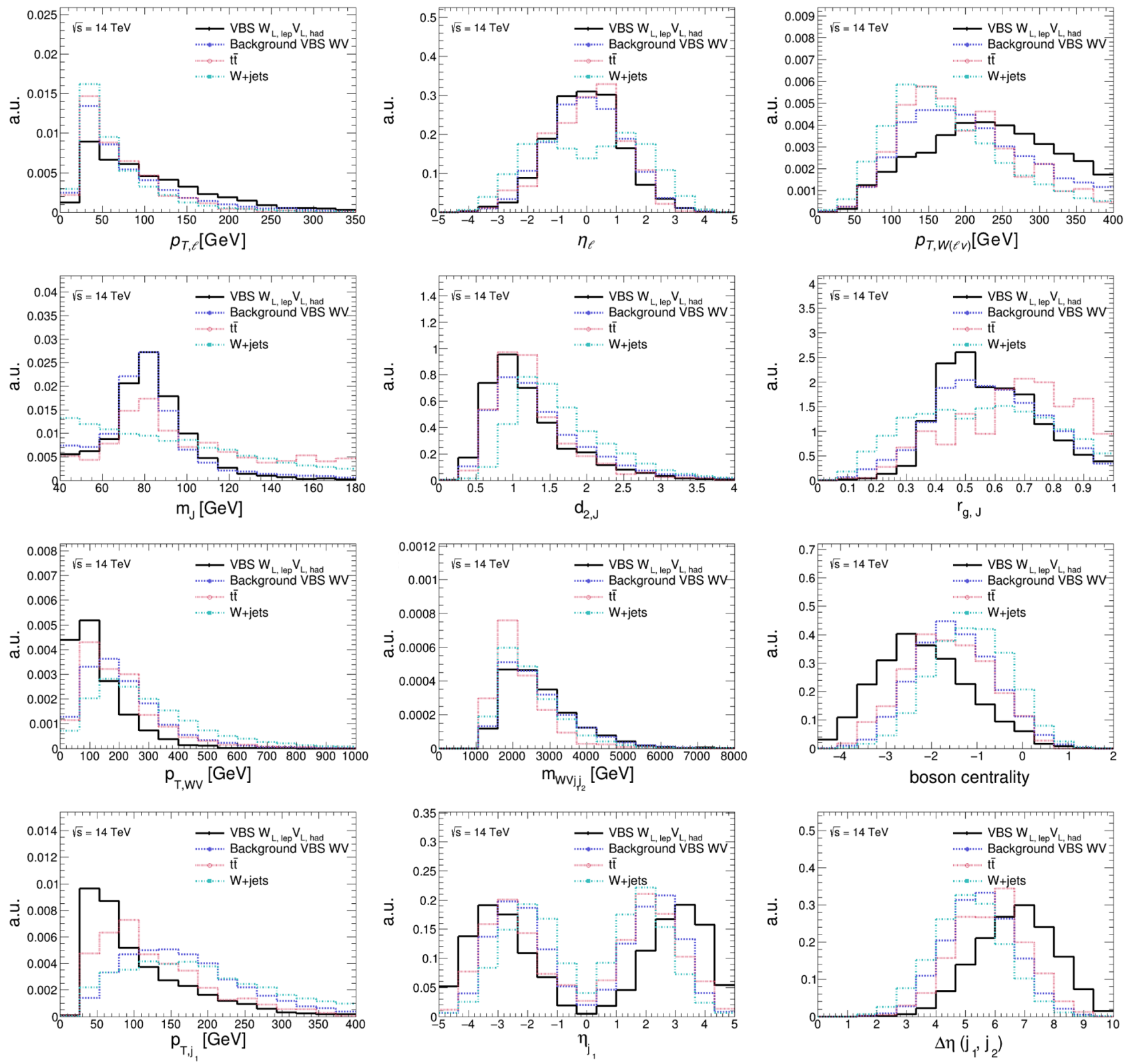

FIG. 5. Distributions of the different inputs to the multiclass tagger in $W+$ jets, top-quark pair production, background VBS $W V$, and signal VBS $W_{L} V_{L}$ events.
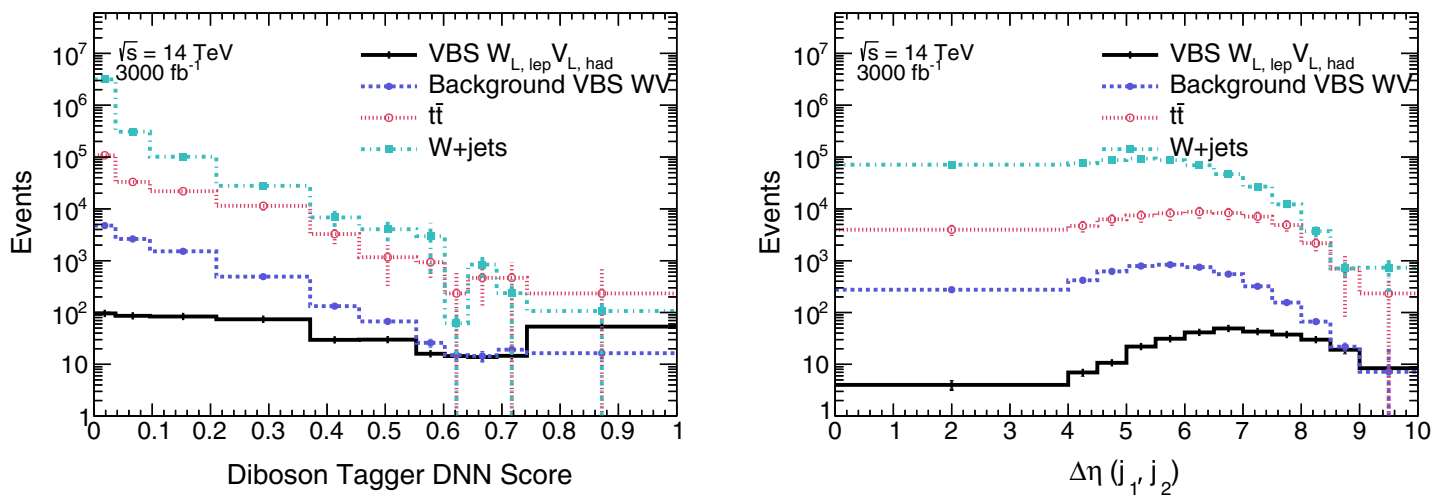

FIG. 6. Signal fit input templates for (left) the multiclass DNN tagger, and (right) $\Delta \eta\left(j_{1}, j_{2}\right)$. The error bars represent the statistical uncertainty. 
background contributions by making full use of the kinematic information available in the event. The output of this DNN tagger for the signal class is used as an input to the template fit utilized to estimate the signal sensitivity, as described in the next section.

\section{ANALYSIS SENSITIVITY}

The analysis sensitivity to the VBS $W_{L} V_{L}$ signal is extracted by performing a simultaneous binned maximumlikelihood fit to the signal and background distributions of the DNN and $\Delta \eta\left(j_{1}, j_{2}\right)$, respectively. A test statistic based on the profile likelihood ratio is used to test hypothesized values of the signal-strength factor. The likelihood is defined as the product of the Poisson likelihood for each bin. The fit includes the main background contributions from $\mathrm{W}+$ jets and top-quark pair production, as well as the background contributions from the different polarization states.

While the sensitivity is limited by the statistical uncertainty, two sources of experimental uncertainties are considered: the jet energy and mass resolution of the large- $\mathrm{R}$ jet. To evaluate these, the energy and mass of the jet are each smeared by $10 \%$. In addition, theoretical normalization uncertainties of $10 \%$ are considered for each background. The normalization uncertainties are found to be dominant over the large-R jet energy and mass uncertainties. Systematic uncertainties are taken into account as constrained nuisance parameters with Gaussian distributions. For each source of systematic uncertainty, the correlations across bins in the distributions and between different kinematic regions as well as those between signal and background are taken into account.

The expected significance is shown in Fig. 7 as a function of the total integrated luminosity, with and without the inclusion of the systematic uncertainties. The total integrated luminosity at the HL-LHC is expected to be $3000 \mathrm{fb}^{-1}$, and our results are shown for up to double this integrated luminosity, giving a simple extrapolation to the expected sensitivity from the combination of measurements from ATLAS and CMS. The sensitivity using the multiclass tagger is compared to using a $\Delta \eta\left(j_{1}, j_{2}\right)$, which shows the best single-variable separation between signal and background. The tagger provides significant gains over the single-variable input, demonstrating the importance of a multivariate tagger to improve the signal significance. The statistical uncertainties are the dominating factor, but some impact from the normalization uncertainties is seen. With the expected luminosity of $3000 \mathrm{fb}^{-1}$ at the HL-LHC, the dataset may be used to separate the longitudinal component of VBS $W V$ with a significance of $3 \sigma$ considering statistical

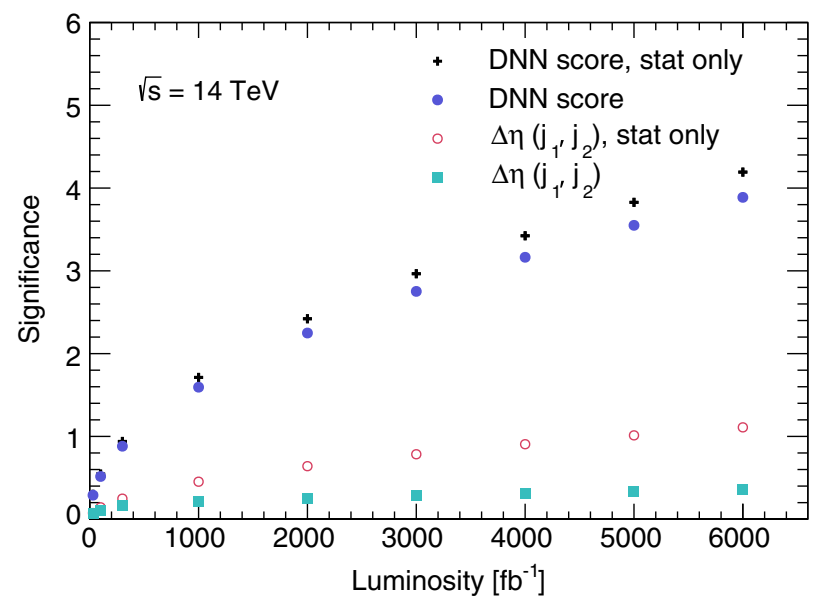

FIG. 7. Expected $W_{L} V_{L}$ significance as a function of the integrated luminosity at the HL-LHC, using the full DNN and the $\Delta \eta\left(j_{1}, j_{2}\right)$ observable alone. The "stat only" results are obtained considering statistical uncertainties only, rather than both statistical and systematic uncertainties.

uncertainties only, and $2.8 \sigma$ when including systematic uncertainties.

\section{SUMMARY AND OUTLOOK}

The measurement of longitudinal VBS production is both a fundamental test of the SM and a window to new physics. We have studied the prospects for measuring longitudinal VBS $W V$ production at the HL-LHC using the semi-leptonic final state where the $V$ boson hadronic decay products are boosted into a single large-radius jet. Using substructure and machine learning techniques, our studies demonstrate that we can expect to establish longitudinal VBS production in this channel with approximately 3 standard deviations at the HL-LHC. Despite the higher backgrounds and more complicated hadronic activity, this sensitivity is similar to what has been projected for the fully leptonic final states. Further improvements may be achieved through the use of the resolved channel, $Z V j j$ semileptonic final states, as well as more complex object reconstruction. Even with the applied restrictions, our study demonstrates the importance of semi-leptonic final states in establishing longitudinal VBS production at the HL-LHC.

\section{ACKNOWLEDGEMENTS}

The work of V. C., M.-A. P. and J. R. is supported by the U.S. Department of Energy, Office of Science, Office of High Energy Physics under Contract No. DE-SC0012704. 
[1] D. A. Dicus, J.F. Gunion, and R. Vega, Isolating the scattering of longitudinal W+'s at the SSC using like-sign dileptons, Phys. Lett. B 258, 475 (1991).

[2] M. J. G. Veltman, Second threshold in weak interactions, Acta Phys. Pol. B 8, 475 (1977), https://inspirehep.net/ literature/110700.

[3] B. W. Lee, C. Quigg, and H. B. Thacker, Strength of Weak Interactions at Very High Energies and the Higgs Boson Mass, Phys. Rev. Lett. 38, 883 (1977).

[4] B. W. Lee, C. Quigg, and H. B. Thacker, Weak interactions at very high energies: The role of the Higgs-boson mass, Phys. Rev. D 16, 1519 (1977).

[5] J. F. Gunion, H. E. Haber, G. L. Kane, and S. Dawson, The Higgs Hunter's Guide, Front. Phys. 80, 1 (2000), https:// inspirehep.net/literature/279039.

[6] G. Aad et al. (ATLAS Collaboration), Observation of a new particle in the search for the Standard Model Higgs boson with the ATLAS detector at the LHC, Phys. Lett. B 716, 1 (2012).

[7] S. Chatrchyan et al. (CMS Collaboration), Observation of a new boson at a mass of $125 \mathrm{GeV}$ with the CMS experiment at the LHC, Phys. Lett. B 716, 30 (2012).

[8] V. Khachatryan et al. (CMS Collaboration), Constraints on the spin-parity and anomalous HVV couplings of the Higgs boson in proton collisions at 7 and $8 \mathrm{TeV}$, Phys. Rev. D 92, 012004 (2015).

[9] V. Khachatryan et al. (CMS Collaboration), Precise determination of the mass of the Higgs boson and tests of compatibility of its couplings with the standard model predictions using proton collisions at 7 and $8 \mathrm{TeV}$, Eur. Phys. J. C 75, 212 (2015).

[10] G. Aad et al. (ATLAS Collaboration), Study of the spin and parity of the Higgs boson in diboson decays with the ATLAS detector, Eur. Phys. J. C 75, 476 (2015).

[11] G. Aad et al. (ATLAS Collaboration), Measurements of the Higgs boson production and decay rates and coupling strengths using $p p$ collision data at $\sqrt{s}=7$ and $8 \mathrm{TeV}$ in the ATLAS experiment, Eur. Phys. J. C 76, 6 (2016).

[12] O. J. P. Eboli, M. C. Gonzalez-Garcia, and J. K. Mizukoshi, $p p \rightarrow j j e^{ \pm} \mu^{ \pm} v v$ and $j j e^{ \pm} \mu^{\mp} v v$ at $O\left(\alpha_{\mathrm{em}}^{6}\right)$ and $O\left(\alpha_{\mathrm{em}}^{4} \alpha_{s}^{2}\right)$ for the study of the quartic electroweak gauge boson vertex at CERN LHC, Phys. Rev. D 74, 073005 (2006).

[13] J. M. Campbell and R. K. Ellis, Higgs constraints from vector boson fusion and scattering, J. High Energy Phys. 04 (2015) 030.

[14] A. Alboteanu, W. Kilian, and J. Reuter, Resonances and unitarity in weak boson scattering at the LHC, J. High Energy Phys. 11 (2008) 010,

[15] S. Godfrey and K. Moats, Exploring Higgs triplet models via vector boson scattering at the LHC, Phys. Rev. D 81, 075026 (2010).

[16] D. Espriu and B. Yencho, Longitudinal $W W$ scattering in light of the "Higgs boson" discovery, Phys. Rev. D 87, 055017 (2013).

[17] J. Chang, K. Cheung, C.-T. Lu, and T.-C. Yuan, WW scattering in the era of post-Higgs-boson discovery, Phys. Rev. D 87, 093005 (2013).

[18] C.-W. Chiang, S. Kanemura, and K. Yagyu, Novel constraint on the parameter space of the Georgi-Machacek model with current LHC data, Phys. Rev. D 90, 115025 (2014).

[19] W. Kilian, T. Ohl, J. Reuter, and M. Sekulla, High-energy vector boson scattering after the Higgs boson discovery, Phys. Rev. D 91, 096007 (2015).

[20] M. Aaboud et al. (ATLAS Collaboration), Observation of electroweak $W^{ \pm} Z$ boson pair production in association with two jets in $p p$ collisions at $\sqrt{s}=13 \mathrm{TeV}$ with the ATLAS detector, Phys. Lett. B 793, 469 (2019).

[21] A. M. Sirunyan et al. (CMS Collaboration), Measurements of production cross sections of WZ and same-sign WW boson pairs in association with two jets in proton-proton collisions at $\sqrt{s}=13 \mathrm{TeV}$, Phys. Lett. B 809, 135710 (2020).

[22] M. Aaboud et al. (ATLAS Collaboration), Observation of Electroweak Production of a Same-Sign $W$ Boson Pair in Association with Two Jets in $p p$ Collisions at $\sqrt{s}=$ $13 \mathrm{TeV}$ with the ATLAS Detector, Phys. Rev. Lett. 123, 161801 (2019).

[23] A. M. Sirunyan et al. (CMS Collaboration), Observation of Electroweak Production of Same-Sign $W$ Boson Pairs in the Two Jet and Two Same-Sign Lepton Final State in ProtonProton Collisions at $\sqrt{s}=13 \mathrm{TeV}$, Phys. Rev. Lett. 120, 081801 (2018).

[24] G. Aad et al. (ATLAS Collaboration), Observation of electroweak production of two jets and a Z-boson pair with the ATLAS detector at the LHC, arXiv:2004.10612.

[25] A. M. Sirunyan et al. (CMS Collaboration), Evidence for electroweak production of four charged leptons and two jets in proton-proton collisions at $\sqrt{s}=13 \mathrm{TeV}$, Phys. Lett. B 812, 135992 (2021).

[26] M. Aaboud et al. (ATLAS Collaboration), Search for anomalous electroweak production of $W W / W Z$ in association with a high-mass dijet system in $p p$ collisions at $\sqrt{s}=8 \mathrm{TeV}$ with the ATLAS detector, Phys. Rev. D 95, 032001 (2017).

[27] A. M. Sirunyan et al. (CMS Collaboration), Search for anomalous electroweak production of vector boson pairs in association with two jets in proton-proton collisions at 13 TeV, Phys. Lett. B 798, 134985 (2019).

[28] G. Aad et al. (ATLAS Collaboration), Search for electroweak diboson production in association with a high-mass dijet system in semileptonic final states in $p p$ collisions at $\sqrt{s}=13 \mathrm{TeV}$ with the ATLAS detector, Phys. Rev. D 100, 032007 (2019).

[29] ATLAS Collaboration, HL-LHC prospects for diboson resonance searches and electroweak vector boson scattering in the $W W / W Z \rightarrow \ell \nu q q$ final state, Report No. ATL-PHYSPUB-2018-022, 2018, https://inspirehep.net/literature/ 1795287.

[30] M. Aaboud et al. (ATLAS Collaboration), Measurement of $W^{ \pm} Z$ production cross sections and gauge boson polarisation in $p p$ collisions at $\sqrt{s}=13 \mathrm{TeV}$ with the ATLAS detector, Eur. Phys. J. C 79, 535 (2019).

[31] A. M. Sirunyan et al. (CMS Collaboration), Measurements of production cross sections of polarized same-sign $\mathrm{W}$ boson pairs in association with two jets in proton-proton collisions at $\sqrt{s}=13 \mathrm{TeV}$, Phys. Lett. B 812, 136018 (2021). 
[32] D. Contardo et al., Technical proposal for the Phase-II upgrade of the CMS detector, Reports No. CERN-LHCC2015-010, No. LHCC-P-008, and No. CMS-TDR-15-02, 2015, https://inspirehep.net/literature/1614097.

[33] CMS Collaboration, Vector Boson Scattering prospective studies in the ZZ fully leptonic decay channel for the High-Luminosity and High-Energy LHC upgrades, Report No. CMS-PAS-FTR-18-014, 2018, https://inspirehep.net/ literature/1708645.

[34] CMS Collaboration, Prospects for the measurement of electroweak and polarized WZ to $31 \mathrm{v}$ production cross sections at the High-Luminosity LHC, Report No. CMSPAS-FTR-18-038, 2018, https://inspirehep.net/literature/ 1708642.

[35] ATLAS Collaboration, Prospective study of vector boson scattering in WZ fully leptonic final state at HL-LHC, Report No. ATL-PHYS-PUB-2018-023, 2018, https:// inspirehep.net/literature/1795274.

[36] CMS Collaboration, Study of $\mathrm{W}^{ \pm} \mathrm{W}^{ \pm}$production via vector boson scattering at the HL-LHC with the upgraded CMS detector, Report No. CMS-PAS-FTR-18-005, 2018, https:// inspirehep.net/literature/1703668.

[37] ATLAS Collaboration, Prospects for the measurement of the $W^{ \pm} W^{ \pm}$scattering cross section and extraction of the longitudinal scattering component in $p p$ collisions at the High-Luminosity LHC with the ATLAS experiment, Report No. ATL-PHYS-PUB-2018-052, 2018, https://inspirehep .net/literature/1795250.

[38] J. Searcy, L. Huang, M.-A. Pleier, and J. Zhu, Determination of the $W W$ polarization fractions in $p p \rightarrow W^{ \pm} W^{ \pm} j j$ using a deep machine learning technique, Phys. Rev. D 93, 094033 (2016).

[39] M. Grossi, J. Novak, B. Kersevan, and D. Rebuzzi, Comparing traditional and deep-learning techniques of kinematic reconstruction for polarization discrimination in vector boson scattering, Eur. Phys. J. C 80, 1144 (2020).

[40] V. Cavaliere, R. Les, T. Nitta, and K. Terashi, HE-LHC prospects for diboson resonance searches and electroweak WW/WZ production via vector boson scattering in the semileptonic final states, arXiv:1812.00841.

[41] J. Alwall, R. Frederix, S. Frixione, V. Hirschi, F. Maltoni, O. Mattelaer, H.-S. Shao, T. Stelzer, P. Torrielli, and M. Zaro, The automated computation of tree-level and next-to-leading order differential cross sections, and their matching to parton shower simulations, J. High Energy Phys. 07 (2014) 079.

[42] T. Sjostrand, S. Mrenna, and P. Z. Skands, A brief introduction to PYTHIA 8.1, Comput. Phys. Commun. 178, 852 (2008).

[43] D. Buarque Franzosi, O. Mattelaer, R. Ruiz, and S. Shil, Automated predictions from polarized matrix elements, J. High Energy Phys. 04 (2020) 082.

[44] S. Catani, F. Krauss, R. Kuhn, and B. R. Webber, QCD matrix elements + parton showers, J. High Energy Phys. 11 (2001) 063.

[45] L. Lonnblad, Correcting the colour-dipole cascade model with fixed order matrix elements, J. High Energy Phys. 05 (2002) 046.

[46] P. Artoisenet, R. Frederix, O. Mattelaer, and R. Rietkerk, Automatic spin-entangled decays of heavy resonances in Monte Carlo simulations, J. High Energy Phys. 03 (2013) 015 .

[47] J. de Favereau, C. Delaere, P. Demin, A. Giammanco, V. Lemaître, A. Mertens, and M. Selvaggi (DELPHES 3 Collaboration), DELPHES 3: A modular framework for fast simulation of a generic collider experiment, J. High Energy Phys. 02 (2014) 057.

[48] P. Azzi et al., Report from Working Group 1: Standard Model physics at the HL-LHC and HE-LHC, CERN Yellow Rep. Monogr. 7, 1 (2019).

[49] ATLAS, CMS Collaborations, Addendum to the report on the physics at the HL-LHC, and perspectives for the HE-LHC: Collection of notes from ATLAS and CMS, CERN Yellow Rep. Monogr. 7 (2019).

[50] M. Cacciari, G. P. Salam, and G. Soyez, FastJet user manual, Eur. Phys. J. C 72, 1896 (2012).

[51] M. Cacciari, G. P. Salam, and G. Soyez, The anti- $k_{t}$ jet clustering algorithm, J. High Energy Phys. 04 (2008) 063.

[52] A. J. Larkoski, S. Marzani, G. Soyez, and J. Thaler, Soft drop, J. High Energy Phys. 05 (2014) 146.

[53] A. J. Larkoski, I. Moult, and D. Neill, Analytic boosted boson discrimination, J. High Energy Phys. 05 (2016) 117.

[54] A. Hoecker, P. Speckmayer, J. Stelzer, J. Therhaag, E. V. Toerne, H. Voss et al., TMVA-toolkit for multivariate data analysis, Report No. CERN-OPEN-2007-007, 2007, https:// inspirehep.net/literature/746087. 